
nature »: Une pratique scientifique au service de l'action (XIX $\mathrm{X}^{\mathrm{e}} \mathrm{XX} \mathrm{X}^{\mathrm{e}}$ siècles)

\title{
Fabien Knitell, Agronomie et innovation, le cas Mathieu de Dombasle (1777-1843)
}

Nancy, Presses Universitaires de Nancy, 2009, 515 p.

\section{Georges Carantino}

\section{(2) OpenEdition}

Journals

Édition électronique

URL : http://journals.openedition.org/dht/1846

DOI : $10.4000 /$ dht. 1846

ISSN : $1775-4194$

Éditeur :

Centre d'histoire des techniques et de l'environnement du Cnam (CDHTE-Cnam), Société des élèves du CDHTE-Cnam

Édition imprimée

Date de publication : 1 décembre 2011

Pagination : 237-239

ISBN : 978-2-9530779-7-1

ISSN : 0417-8726

Référence électronique

Georges Carantino, «Fabien Knitell, Agronomie et innovation, le cas Mathieu de Dombasle

(1777-1843) », Documents pour l'histoire des techniques [En ligne], 20 | 2e semestre 2011, mis en ligne le 24 septembre 2012, consulté le 24 septembre 2020. URL : http://journals.openedition.org/dht/1846 ;

DOI : https://doi.org/10.4000/dht.1846

Ce document a été généré automatiquement le 24 septembre 2020

(c) Tous droits réservés 


\section{Fabien Knitell, Agronomie et innovation, le cas Mathieu de Dombasle (1777-1843)}

Nancy, Presses Universitaires de Nancy, 2009, 515 p.

\section{Georges Carantino}

\section{RÉFÉRENCE}

Fabien Knitell, Agronomie et innovation, le cas Mathieu de Dombasle (1777-1843), Nancy, Presses Universitaires de Nancy, 2009, 515 p. 
1
Cet ouvrage est la publication d'une thèse soutenue à l'Université Nancy 2 en juillet 2007 qui a obtenu la mention très honorable et les félicitations du jury, thèse récompensée d'un Prix de Thèse par le Conseil Régional de Lorraine pour cette restitution de tout un pan mal connu de l'histoire de la région. L'auteur se propose de relire la figure de Mathieu de Dombasle, agronome lorrain du début du XIXe siècle sur lequel beaucoup d'ouvrages ont paru relevant souvent d'une vision de l'«inventeur-héros » ou de l'hagiographie. Aux yeux de

l'historiographie, Mathieu de Dombasle apparait comme le concepteur d'une charrue innovante et le fondateur du premier établissement d'enseignement agricole en France. Fabien Knittel veut plutôt étudier la trajectoire d'un homme à un moment charnière dans l'histoire de l'agronomie, discipline scientifique alors en pleine constitution, passant d'une agronomie-art à une agronomie-science, première phase de son institutionnalisation qui s'étend de 1750 aux années 1850, de la floraison des écrits agronomiques à l'affirmation de la chimie agricole. Il veut le voir comme un acteur majeur tant régional que national en lien avec une configuration sociologique particulière, au sens de Norbert Elias, configuration faite d'une volonté largement partagée de modernisation de l'agriculture marquée par l'influence des physiocrates, du rôle important de l'Etat relayé par l'action des Sociétés d'Agriculture, modernisation portée par l'action conjuguée de la mécanisation de l'agriculture et du développement de l'enseignement. Fabien Knittel veut ramener Mathieu de Dombasle à ce qu'il est, un « agronome ordinaire » dont la charrue est une « pseudo-invention ». Il repense ainsi le genre biographique en valorisant les logiques collectives au détriment de la figure de l'« inventeur-héros ». Cette thèse n'est pas seulement un travail d'historien, l'auteur prend une position transdisciplinaire. Les concepts de l'agronomie actuelle sont utilisés comme «supports réflexifs » pour mieux comprendre les données historiques, écrits des agronomes contemporains de Mathieu de Dombasle, écrits et démarche de Mathieu de Dombasle lui-même. Aussi se réfère-t-il souvent à de nombreux agronomes actuels, Gilles Denis, Paul Robin, Jean Boulaine, Stéphane Hénin... Les concepts agronomiques de "système de culture ", de "profil cultural ", le concept d' "itinéraire technique " ont été des clés pour analyser la démarche de Mathieu de Dombasle. « La connaissance par l'historien de la théorie du profil cultural lui facilite la compréhension des démarches techniques mises en œuvre par des agronomes ou des paysans à une époque donnée » écrit-il page 101. Et il se plait à citer Marc Bloch dans «Apologie pour l'histoire »: " Les documents tendent à imposer leur nomenclature. L'historien, s'il les écoute, écrit sous la dictée d'une époque chaque fois différente. Mais il pense, d'autre part, naturellement selon les catégories de son propre temps; par suite avec les mots de celui-ci ». Fabien Knittel fait aussi appel aux concepts de la sociologie et cite souvent Norbert Elias, Pierre Bourdieu... Il s'interroge sur la nature de l'invention dans son souci de déconstruire l'image d'« inventeur-héros » que l'historiographie traditionnelle a associée à Mathieu de Dombasle. Pour ce il ressent le besoin de s'appuyer sur des auteurs reconnus comme A.G. Haudricourt et M. Jean-Brunhes Delamare qu'il cite 
abondamment page 108: "L'esprit humain n'invente rien ex-nihilo, "invention ", "naissance " signifient en réalité recombinaison d'éléments qui aboutissent à du «nouveau », à de l' " original ", non pas surgi du vide, mais résultat d'un faisceau de facteurs longuement préparés et d'un entrelacs d'actes et de réflexions... L'invention étant l'aboutissement d'un corps existant de connaissances, comment attribuer une invention à un seul inventeur?».

2 La charrue « Dombasle ", qui n'a pas d'avant-train et est équipée d'un régulateur, n'est donc pas réellement, pour l'auteur, une invention mais une innovation, c'est-à-dire l'adoption d'une nouveauté. Et il montre que cette charrue procède à la fois de la recombinaison d'éléments observés sur la charrue lorraine traditionnelle et sur des modèles anglais mis au point dès les XIIIe et XIVe siècles et de la réflexion et des expériences d'agronomes anglais et allemands de la seconde moitié du XVIIIe siècle, comme Small, Thaër, Fellemberg, Sinclair dont Mathieu de Dombasle connait et a traduit les écrits. Aussi Fabien Knittel ne reconnait-il à Mathieu de Dombasle, en matière de machinisme agricole, qu'un statut de continuateur héritier de l'agronomie des Lumières et de la Physiocratie.

3 Pour notre auteur l'aspect novateur de l'œuvre de Mathieu de Dombasle réside dans son rôle prédominant dans l'institutionnalisation de l'agronomie durant la période 1820-1850, qu'il s'agisse de son rôle à la Société Centrale d'Agriculture de Nancy, de la gestion de la ferme exemplaire de Roville-devant-Bayon dans la Meurthe où la réflexion se conduit selon trois axes, succession culturale, itinéraires techniques et organisation du travail hiérarchisée. Qu'il s'agisse aussi de la mise en place de moyens de diffusion de l'innovation comme la fabrique d'instruments aratoires de Roville organisée selon les principes de la rationalisation du travail et de la standardisation de la production, d'organisation de réunions de praticiens agricoles de la région, bien avant que ne se créent les Comices Agricoles, réunions consistant en démonstrations de machines, en concours de charrue comme en ventes d'animaux ou de la création, en 1826, à Roville, de l'Institut Agricole, premier institution d'enseignement agricole en France, à destination des futurs chefs de domaines. Le lien entre théorie et pratique, manifeste dans la démarche scientifique et technique de Mathieu de Dombasle, se retrouve dans sa conception de l'enseignement. Son enseignement est d'abord fondé sur la «clinique agricole ", une pratique basée sur l'observation et l'expérience, sur des conférences en plein air lors du «tour de plaine ». Mais il ne néglige pas l'importance des cours théoriques et celle de la connaissance livresque qui est toujours pour lui la forme essentielle de la diffusion des connaissances et du progrès agricole. Son «Calendrier du bon cultivateur ou manuel de l'agriculteur praticien » et ses «Annales agricoles de Roville » en huit tomes l'attestent.

4 Cet ouvrage est construit en quatre parties qui présentent les grandes étapes du cheminement de Mathieu de Dombasle, les «configurations " à l'échelon national et régional dans lesquelles il se situe, son rôle dans l'institutionnalisation de l'agronomie en Lorraine et les moyens de diffusion qu'il met en place. Dans la première partie de l'ouvrage, l'auteur, après avoir longuement réfléchi sur la démarche biographique, sur des questions de vocabulaire comme le sens des termes " agronome » et " agronomie ", sur la théorie de l'innovation et sur les concepts utiles à l'historien dans ce champ de recherche, évoque Mathieu de Dombasle comme un jeune noble féru de sciences puis d'agriculture. Il parle de ses premiers travaux sur la chimie de l'eau puis il montre comment il passe de la chimie à l'agronomie en devenant producteur de sucre donc 
cultivateur de betteraves sur son domaine de Monplaisir. La culture de la betterave à sucre le confronte aux problèmes du travail du sol qui le conduisent à une réflexion technique sur les labours et les types de charrues. Difficultés économiques et faillite l'éloigneront de la culture de la betterave et de la production du sucre dont il était devenu un expert par des écrits remarqués. Il rentrera alors en agronomie par le travail du sol et se consacrera à une "théorie de la charrue ». Cette théorie le conduira à innover dans une charrue sans avant-train que l'on nommera "charrue Dombasle », et ses réflexions le mèneront à la publication, en 1821, des "Mémoires sur la charrue considérée principalement sous le rapport de la présence ou de l'absence de l'avanttrain » qui lui vaudront une audience nationale. Son souci est d'obtenir « les labours les plus parfaits avec autant d'économie qu'il est possible ». Ce qu'il réussira car sa charrue nécessite un attelage restreint et une main d'œuvre réduite. Récompenses et reconnaissance suivront.

5 La deuxième partie de l'ouvrage veut cerner les fondements de la pensée agronomique de Mathieu de Dombasle. Elle présente d'abord la configuration globale dans laquelle il évolue pour essayer de cerner son " outillage mental ", évoquant l'agriculture nouvelle dans la seconde moitié du XVIIIe siècle, l'émergence de l'agronomie et la pensée physiocratique, évoquant aussi Mathieu de Dombasle comme lecteur et traducteur d'écrits agronomiques étrangers. Elle présente aussi la configuration lorraine, entre progrès et frein à l'innovation, par l'évocation des pratiques paysannes en particulier dans les Vosges, par l'intérêt des grands pour la nouveauté, par la diffusion du progrès agronomique en Lorraine avant 1820, par l'intérêt local pour le labour et le travail du sol. Cette deuxième partie évoque aussi les conditions culturelles favorables qui s'offrent à Mathieu de Dombasle: les premières leçons d'économie rurale dans les écoles vétérinaires, le projet de l'Abbé Grégoire qui, préoccupé par la diffusion des techniques culturales innovantes et par l'enseignement de l'agriculture, soumet à la Convention un rapport sur la création de "maisons d'économie rurale » dans chaque département, les propositions de François de Neufchâteau de créer une grande école d'économie rurale et l'exemple d'Antoine Bertier qui l'a précédé à Roville où il crée une école d'enseignement mutuel agricole.

6 Dans une troisième partie l'auteur évoque la contribution de Mathieu de Dombasle à l'institutionnalisation de l'agronomie dans sa province. Il présente son engagement dans les sociétés savantes lorraines en particulier la Société Royale des Sciences et Belles Lettres de Nancy qui se préoccupe d'agronomie et d'agriculture, son implication dans la création de la Société Centrale d'Agriculture de Nancy et son souci de moderniser l'agriculture locale par des concours, des essais et par l'enseignement. L'auteur présente ensuite la ferme exemplaire de Roville, sa création comme établissement agricole d'un type nouveau en s'appuyant sur un bail modèle et le réseau agronomique européen qui se tisse autour d'elle. Il la décrit et présente les systèmes de culture qu'elle met en place. Il montre bien qu'autour de cette ferme se crée tout un mouvement d'émulation qui pousse un modeste valet de ferme, Jean Joseph Grangé, à concevoir et à diffuser un nouveau modèle de charrue, la charrue "Grangé ", comme elle stimule l'œuvre d'agronome de Louis Poirot de Valcourt, « agronome dans l'ombre de Mathieu de Dombasle ».

7 La dernière partie est consacrée aux moyens de diffusion de l'innovation que met en place, à Roville, Mathieu de Dombasle. L'auteur évoque d'abord la «fabrique d'instruments aratoires perfectionnés » et présente son fonctionnement. C'est le lieu de 
fabrication, d'amélioration et de diffusion de la charrue "Dombasle ». C'est autour de cette fabrique que s'organisent les concours de labours, « une pratique de diffusion innovante ", entre émulation et vulgarisation. La mise en place des comices agricoles sera la raison de l'abandon de ces concours à Roville. Puis est présenté l'Institut Agricole destiné à former des chefs de domaine. C'est une initiative fondée davantage sur l'exemple germanique qu'anglais. Est alors détaillée la pédagogie de l'établissement fondée sur la clinique agricole mais aussi sur les enseignements théoriques et une transmission par l'écrit. L'étude "socio-historique " des élèves montre qu'au bout de quelques années le recrutement se fait à l'échelon national. D'où l'intérêt du dernier point abordé, l'influence de la pédagogie de Mathieu de Dombasle et ses héritages. Si les élèves ont été nombreux durant les vingt ans qu'a duré cette formation, la réussite semble nuancée quant à la formation des chefs de domaine. La vraie réussite de cette innovation dans la diffusion des savoirs est que Roville a été le lieu de la formation des cadres de l'enseignement agricole français qui va se mettre en place à l'échelle du territoire français à partir du milieu du XIXe siècle.

La conclusion de l'auteur reprend une longue réflexion sur la conception de la biographie qu'il a voulu mettre en œuvre, sur les limites de ce que l'on peut connaitre de Mathieu de Dombasle et sur les pistes de recherche future que son travail a ouvertes. Saluons la présence en fin de volume d'une longue liste de sources consultées et en particulier de l'inventaire des écrits de Mathieu de Dombasle, ainsi que d'une importante bibliographie. Saluons aussi la présence d'un "lexique sommaire des termes du travail du sol ». La présence d'index aurait été commode. On aura compris à la lecture de ce bref compte-rendu que nous avons affaire à un ouvrage riche d'une information dense et d'une importante réflexion de méthode. On regrettera cependant que l'envahissant besoin de fonder sa pensée par un recourt fréquent à des auteurs qui font référence, ai conduit l'auteur à truffer souvent son texte de citations qui nuisent à la fluidité de la lecture et la rende parfois pénible. La rédaction de notes plus fournies aurait pu y remédier. 